\title{
A Dynamic Semi-Circular Bending Configuration for the Rheological Characterization of Asphalt Mixtures
}

\author{
Marina Cauhape Casaux, Silvia Angelone, Fernando Martinez \\ Road Laboratory, Institute of Applied Mechanics and Structures, National University of Rosario, Riobamba \& \\ Berutti, (2000) Rosario, Argentina \\ E-mail:fermar@fceia.unr.edu.ar
}

Received: 5 June 2020; Accepted: 7 July 2020; Available online: 30 July 2020

\begin{abstract}
Many experimental procedures have been proposed for the determination of the rheological properties of asphalt mixtures. Among them, the indirect tensile test (ITT) has gained a lot of attention because of its relative simplicity and advantages. However, a biaxial state of stress is developed into the sample and then, the Poisson ratio must be estimated or measured for the calculations of the dynamic modulus. Looking for a testing configuration with the same simplicity and advantages, this paper proposes the Semi-Circular Bending (SCB) geometry with dynamic loading conditions in order to characterize the rheological properties (dynamic modulus and phase angle) of asphalt mixtures. Samples compacted in the laboratory or cored from in-service pavements can be used. A uniaxial state of stress is developed in the lower plane surface of the sample doing negligible the influence of the Poisson ratio on the dynamic modulus calculations. A specific experimental configuration was adopted and a 2D-FEM model has been used for the development of an equation for the calculation of the dynamic modulus. Two different asphalt mixtures were tested at diverse testing temperatures and loading frequencies. The obtained results have been analyzed and compared with those acquired with the more conventional testing configuration in uniaxial compression. An excellent agreement has been found for either the dynamic modulus or the phase angle with both sets of results. It could be concluded that the SCB configuration has been validated as a simple and promising methodology for the characterization of the viscoelastic response of asphalt mixtures.
\end{abstract}

Keywords: Rheological properties; Asphalt mixtures; Semi-circular bending test.

\section{Introduction}

The rheological characteristics of asphalt mixtures have been traditionally determined in laboratory by different experimental procedures evaluating the viscoelastic properties (dynamic modulus and phase angle) under different conditions of loading frequencies and testing temperatures. Among others, dynamic uniaxial and triaxial compression tests, tension-compression tests, indirect tensile tests, two and four point bending tests were extensively used by different researchers.

In this paper the use of the Semi-Circular Bending (SCB) test configuration for the characterization of the rheological properties of asphalt mixtures is investigated. This configuration was selected because it is indicated that the SCB test possesses several advantages as the test setup is simple and virtually any loading frame used with other testing configuration can be adapted for the test, the used specimens can be prepared by sawing samples fabricated in the laboratory or cored in the field from in service pavements.

Also, the required loads to obtain measurable deformations into the specimen are lower than those required by other tests.

The SCB test was initially used to evaluate the fracture resistance in rock mechanics [1, 2]. Later, the SCB test was applied on asphalt mixtures to evaluate the fatigue properties [3] and the tensile strength [4]. Mull et al [5] have used the SCB configuration to evaluate the tensile strength on notched specimens to evaluate the fracture resistance of asphalt mixtures.

Molenaar et al [6] have investigated the fracture resistance of asphalt mixtures and they concluded that the SCB is a simple, low cost test that easily can be performed on specimens prepared by means of the gyratory compactor or on specimens taken from roads.

More recently many studies have been focused on the SCB test as a simple methodology to characterize the fracture and fatigue properties of asphalt mixtures [7-15].

The European Standard EN 12697-44 [16], the American Standard ASTM D8044 [17] and the AASHTO TP 105-13 [18] describe the procedure to evaluate the fracture and crack propagation properties of asphalt mixtures 
using the SCB test. Under dynamic conditions, the SCB test with haversine loads was used to analyze the relationship between the fatigue and crack propagation properties of asphalt mixtures [19].

While the SCB test was used to characterize the fracture and fatigue properties of asphalt mixtures there are not references for the application of the same testing configuration for the rheological characterization of this type of materials through the determination of the Dynamic modulus $\left|\mathrm{E}^{*}\right|$ and the Phase angle $\phi$.

So, the main objective of this paper is the evaluation of the SCB configuration as a potential testing procedure to measure the rheological behavior of bituminous mixtures quantifying the Dynamic modulus $\left|E^{*}\right|$ and Phase angle $\phi$ as two fundamental material parameters characterizing the viscoelastic properties of them. The basic hypothesis behind this investigation is that, if $\left|\mathrm{E}^{*}\right|$ and $\phi$ are considered fundamental material properties, they could be determined using any experimental procedure taking into account the adequate theoretical basis and calculations. Two different asphalt mixtures formulated with two types of asphalt binder were used in this study. Furthermore, the conventional uniaxial test in compression was also conducted to validate the reliability of the SCB configuration for the proposed objective.

\section{Discussion of the SCB test}

Several experimental configurations have been proposed for the rheological characterization of asphalt mixtures. For example, the standard AASHTO T 342-11 [20] describes the procedures for preparing and testing asphalt concrete mixtures using laboratory-prepared specimens in uniaxial compression condition under sinusoidal loads at different frequencies and temperatures. For this testing configuration, specimens with $100 \mathrm{~mm}$ diameter and 150 $\mathrm{mm}$ tall are used. However, the thickness of a typical asphalt pavement layer is only few centimeters. As a consequence, the required specimens cannot be directly cored from the surface of the pavement and hence, this experimental configuration seems not to be adequate for forensic studies of existing pavements.

Based on the hypothesis that the Dynamic Modulus $\left|\mathrm{E}^{*}\right|$ is a true material property describing the stiffness properties of the asphalt mixtures, it could be concluded that any experimental procedure can be adopted considering the adequate theoretical basis and calculations.

In a previous paper, the determination of the dynamic modulus $\left|\mathrm{E}^{*}\right|$ using the Indirect Tensile Test configuration (ITT) with haversine loads at different temperatures and frequencies was validated for asphalt mixtures commonly used in Argentina [21]. In that paper, the equation for the calculation of $\left|E^{*}\right|$ with the ITT configuration was developed following the same procedure as it was presented by other authors $[22,23]$. The resulting equation was:

$$
\lfloor E *\rfloor=\frac{P_{0}}{\Delta h_{o} \cdot h} \cdot\left(K_{1}+\mu \cdot K_{2}\right)
$$

where $\mathrm{P}_{0}$ is the amplitude of the applied haversine load, $\Delta \mathrm{h}_{0}$ is the amplitude of the resulting horizontal deformation, $\mathrm{K}_{1}$ and $\mathrm{K}_{2}$ are coefficients depending on the specimen diameter and gauge length and $\mu$ is the Poisson's ratio.

This experimental procedure was standardized in the European Standard EN 12697-26 - Annex F [24] and in the German Standard AL-SP - Asphalt 09 [25].

As can be observed, the calculation of $\left|E^{*}\right|$ is affected by the Poisson's ratio $\mu$ and then, if only the horizontal deformation is measured, this Poisson's ratio must be adopted or assumed. In some cases, a constant value equal to 0.35 is usually recommended.

In other cases, the Poisson's ratio can be adopted by using a relationship between $\mu$ and the testing temperature $\mathrm{T}$ as it was proposed by Witczak and Mirza [26]:

$$
\mu=0.15+\frac{0.35}{1+e^{\left[3.1849-0.04233 \cdot\left(9 / 5^{T+32}\right)\right]}}
$$

It could be demonstrated that the influence of the Poisson's ratio on the calculation of the dynamic modulus value is very significant and differences greater than $25 \%$ could be obtained if different Poisson's ratio are used.

Also in the ITT configuration, the sample is under a biaxial state of stress where the relationship between the vertical and the horizontal stress components is constant to a fixed value equal to 3 .

In contrast, Molenaar et at. [6] have stated that the SCB is a simple, low cost test that easily can be performed on specimens prepared by means of a gyratory compactor or on specimens cored from the road. Also, the SCB has the big advantage over the well-known ITT test being the fact that the specimens fail in a way indicating that tension might be the dominant failure mode even at higher temperatures. This is contrary to the type of failure often observed with the ITT tests which indicates that the specimens fail due to a mixed mode of stress conditions. 
Thus, an advantage of the SCB configuration is that the stress in the lower plane surface of the semi-circular specimen is a uniaxial tensile stress, and hence, it could be demonstrated that the influence of the Poisson's ratio on the calculation of $\left|E^{*}\right|$ could be considered as negligible.

Also, the test setup is simple and virtually any loading frame used with other testing configuration can be adapted for the test. Finally, the required loads to obtain measurable deformations are lower than those required by other tests and hence, the induced damage into de specimen is minimized.

However, a disadvantage of the SCB is that there is not an analytical solution available for the stress distribution into the specimen and the required equation necessary for the calculation of the dynamic modulus $\left|\mathrm{E}^{*}\right|$ must be developed using a Finite Element model.

Based on this analysis, the SCB in dynamic conditions using haversine loads was investigated as a potential test for the rheological characterization of asphalt mixtures.

\section{Adopted SCB configuration}

\subsection{SCB geometry}

The adopted Semi-Circular Bending configuration is shown in Figure 1.

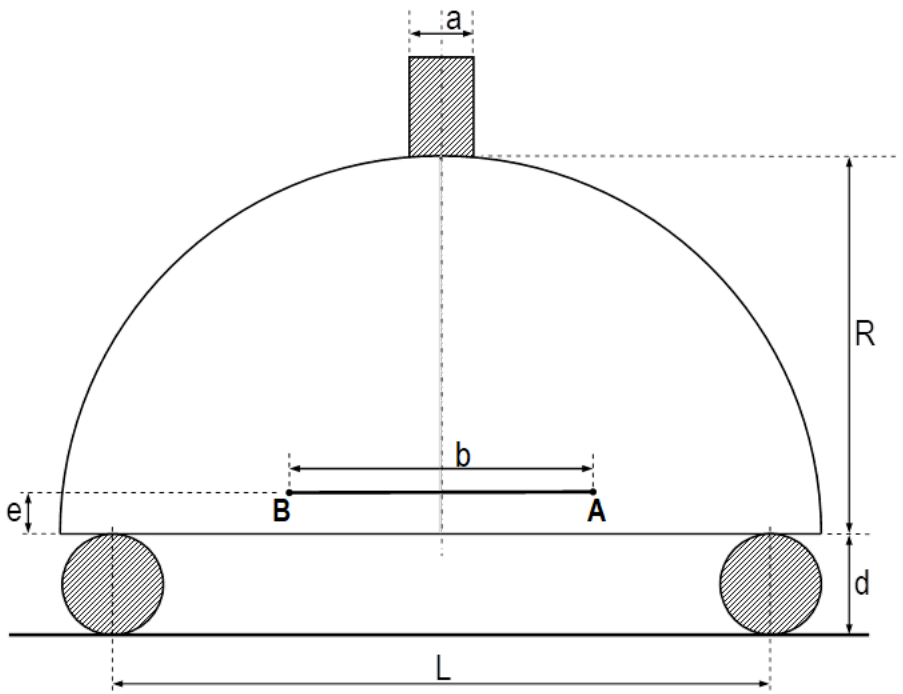

Figure 1. Adopted SCB configuration

For this adopted configuration, $\mathrm{R}$ is the radio of the sample $(\mathrm{R}=75 \mathrm{~mm})$, $\mathrm{d}$ is the diameter of the rollers $(\mathrm{d}=$ $20 \mathrm{~mm}$ ), $\mathrm{L}$ is the spacing between rollers $(\mathrm{L}=130 \mathrm{~mm}), \mathrm{b}$ is the gauge length between the gauge points $\mathrm{A}$ and $\mathrm{B}$ $(\mathrm{b}=60 \mathrm{~mm})$, e is the vertical location of the LVDT`s $(\mathrm{e}=8 \mathrm{~mm})$ and $\mathrm{a}$ is the thickness of the loading strip (a= $12,7 \mathrm{~mm})$.

The specimen is un-notched and a pair of LVDT's is attached along the gauge length on both semi-circular flat faces in order to measure the resulting deformations. Figure 2 shows the experimental setup according to this adopted SCB configuration.

\subsection{Calculation of the dynamic modulus}

There is not an analytical solution based on the applied mechanics available for the calculation of the stress distribution into the specimen for the adopted SCB configuration and consequently, for the calculation of the dynamic modulus $\left|E^{*}\right|$.

Molenaar et al. [6] stated that the modulus of the material can be determined following the usual frequency/temperature sweep and then, the resilient modulus value characterizing the viscoelastic properties of the asphalt mixtures can be calculated using the following equation.

$$
M r=1.84 \mathrm{~F} / \mathrm{V}
$$

where $\mathrm{Mr}$ is the resilient modulus, $\mathrm{F}$ is the load per unit width of the specimen at failure, and $\mathrm{V}$ is the vertical deformation. It should be noted that the equation is valid only if the spacing between the rollers equals 1.6 R.

Using a finite element analysis, Huang et al. [27] have proposed that a stiffness modulus E can be calculated as: 


$$
E=1.997 \cdot\left[e^{1.175^{L} /(2 R)}-1\right] \cdot \frac{P}{d}
$$

where $\mathrm{d}$ is the vertical deflection at the middle point of the lower surface of the specimen and $\mathrm{P}$ is the applied load.

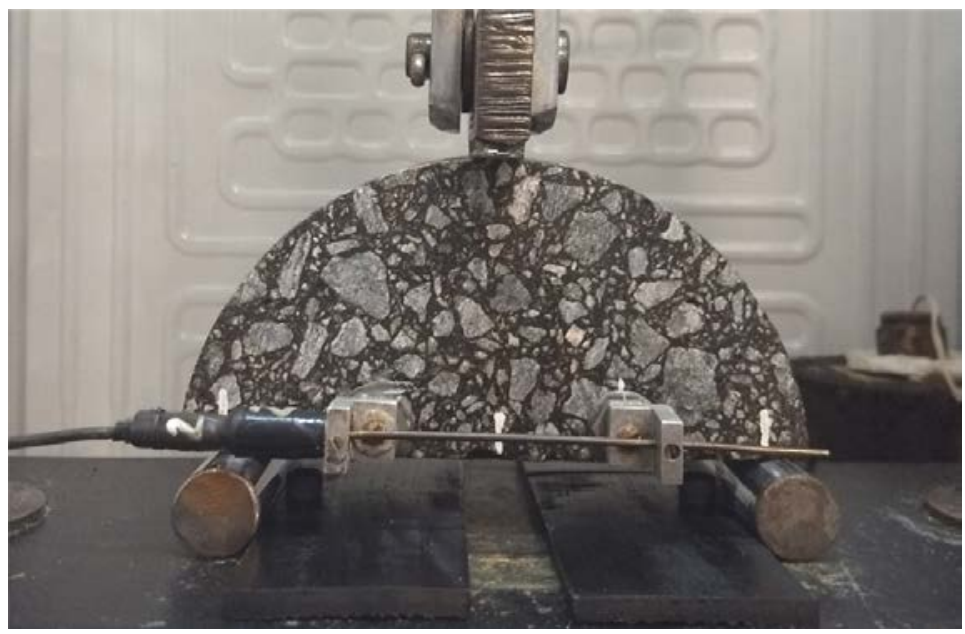

Figure 2. Experimental setup for the SCB test

Saha and Biligiri [19] have conducted fatigue tests using the SCB geometry and they proposed that the viscoelastic characterization of the mixtures can be obtained through the dynamic modulus and the phase angle. The dynamic modulus ESCB can be measured as a ratio of the horizontal tensile stress $\sigma$ to the tensile strain $\varepsilon$ t. The tensile stress $\sigma \mathrm{t}$ due to the vertical load is calculated as:

$$
\sigma t=\frac{P}{D . T}
$$

where $\mathrm{P}$ is the vertical load, $\mathrm{D}$ is the specimen diameter and $\mathrm{T}$ is the specimen thickness.

The tensile strain $\varepsilon t$ is calculated using the Crack Mouth Opening Displacement (CMOD) as:

$$
\varepsilon t=\frac{C M O D}{D}
$$

and the phase angle $\phi_{\mathrm{SCB}}$ is obtained as:

$$
\emptyset_{S C B}=\frac{t i}{t p} \cdot 360^{\circ}
$$

where $t i$ is the time lag between a cycle of stress and strain and $t p$ is the time for a stress cycle.

In this study, a Finite Element Model (FEM) was used in order to develop an equation for the calculation of the dynamic modulus $\left|E^{*}\right|$. For this preliminary evaluation of the SCB geometry and for simplicity of treatment, some assumptions were introduced in the analysis. A 2D finite element model developed at the Road Laboratory of the University of Rosario was used [28]; the material of the specimen was considered as linear, elastic, and isotropic; the specimen was in the plane stress state; and the loading rollers were rigid. Figure 3 shows the finite element mesh used in the analysis and the location of the gauge points A and B.

Based on the results obtained with the FEM and according to the elastic-viscoelastic correspondence principle applied to the SCB geometry with sinusoidal loads in a steady state, the dynamic modulus $\left|E^{*}\right|$ is calculated as:

$$
\lfloor E *\rfloor=1237 \cdot \frac{P_{0}}{\Delta u_{0} \cdot h}
$$

where $\left|\mathrm{E}^{*}\right|$ is the dynamic modulus $(\mathrm{MPa}), \mathrm{P}_{0}$ is the amplitude of the applied sinusoidal load $(\mathrm{N}), \Delta \mathrm{u}_{0}$ is the amplitude of the resulting horizontal deformation of the gauge length $\left(10^{-6} \mathrm{~m}\right)$ and $\mathrm{t}$ is the thickness of the sample (m) as it is shown in Figure 4.

The phase angle $\phi$ between load and deformation is calculated according to: 


$$
\phi=\frac{t}{\tau} \cdot 360^{\mathrm{o}}
$$

where $\phi$ is the phase angle, $\mathrm{t}$ is the time lag between a cycle of deformation and load and $\tau$ is the period for a load cycle.

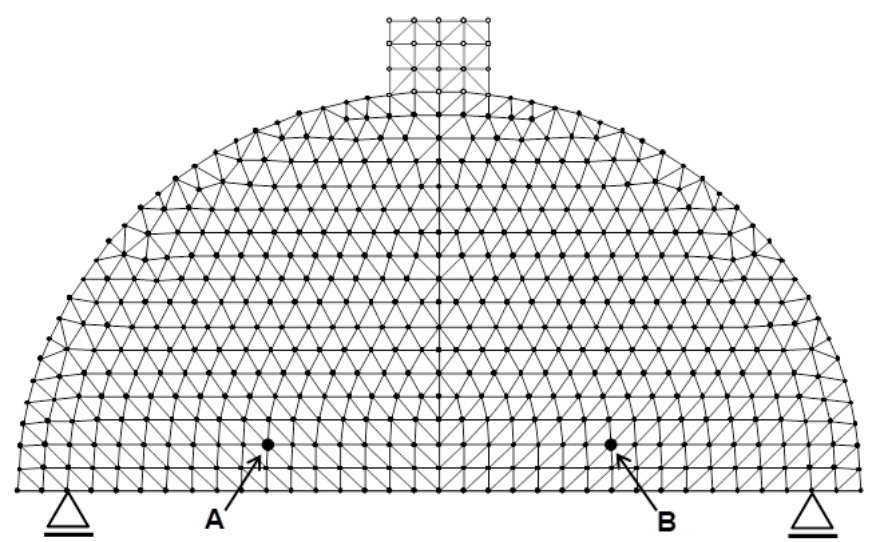

Figure 3. Finite element model

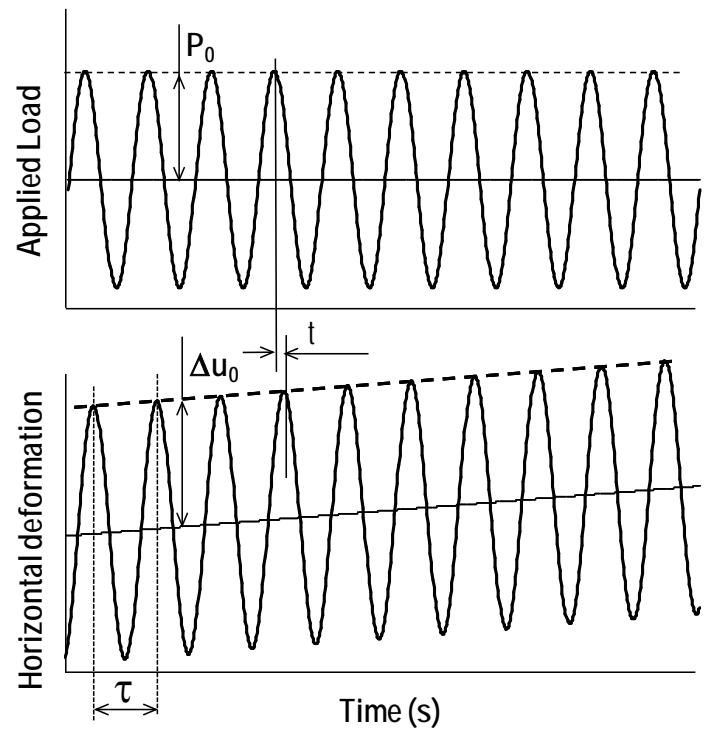

Figure 4. Applied load and resulting horizontal deformation of the gauge length

\section{Materials and procedures}

\subsection{Asphalt mixtures}

Two dense asphalt concretes commonly used in Argentina for base courses were considered in this study in order to validate the SCB geometry for the determination of the viscoelastic characteristics of these materials. One of them, identified as AC30, was formulated with a conventional asphalt binder while the other, named as AM3, was elaborated with a SBS polymer modified asphalt binder. The main volumetric and mechanical properties of these mixtures are listed in Table 1 while the aggregate gradation is listed in Table 2.

\subsection{Sample preparation and testing conditions}

Samples with $150 \mathrm{~mm}$ diameter by $150 \mathrm{~mm}$ height were compacted by gyratory compaction and then, specimens with $100 \mathrm{~mm}$ diameter were cored as shown in Figure 5(a) to be used in the dynamic modulus tests in axial compression. Also, slices with $50 \mathrm{~mm}$ height were sawn and cut as shown in Figure 5(b) to produce semi-circular specimens to be used in the dynamic modulus tests with the SCB configuration. 
Table 1. Volumetric and mechanical properties of the tested asphalt mixtures

\begin{tabular}{lcc}
\hline Property & AC30 & AM3 \\
\hline Asphalt Content by Weight AC (\%) & 4.7 & 4.7 \\
Asphalt Content by Volume Vb (\%) & 11.6 & 11.5 \\
Air Voids Content Va (\%) & 5.1 & 4.9 \\
Theoretical Max. Spec. Gravity Gmm $\left(\mathrm{kg} / \mathrm{dm}^{3}\right)$ & 2.597 & 2.588 \\
Bulk Specific Gravity Gmb $\left(\mathrm{kg} / \mathrm{dm}^{3}\right)$ & 2.464 & 2.460 \\
Voids in the mineral aggregate VMA (\%) & 16.7 & 16.4 \\
Voids filled with asphalt VFA (\%) & 69.5 & 70.1 \\
Marshall Stability (kN) & 15.0 & 13.6 \\
Marshall Flow (mm) & 4.1 & 4.8 \\
\hline
\end{tabular}

Table 2. Aggregate gradation of the asphalt mixtures

\begin{tabular}{lc}
\hline Sieve size & Percent Passing (\%) \\
\hline $25.4 \mathrm{~mm}$ & 100 \\
$19 \mathrm{~mm}$ & 99.6 \\
$9.5 \mathrm{~mm}$ & 73.6 \\
$4.75 \mathrm{~mm}$ & 47.7 \\
$2.36 \mathrm{~mm}$ & 33.2 \\
$0.6 \mathrm{~mm}$ & 18.8 \\
$0.3 \mathrm{~mm}$ & 15.0 \\
$0.075 \mathrm{~mm}$ & 8.0 \\
\hline
\end{tabular}

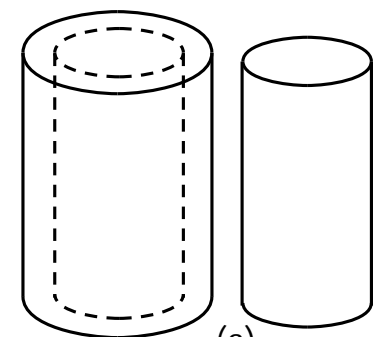

(a)

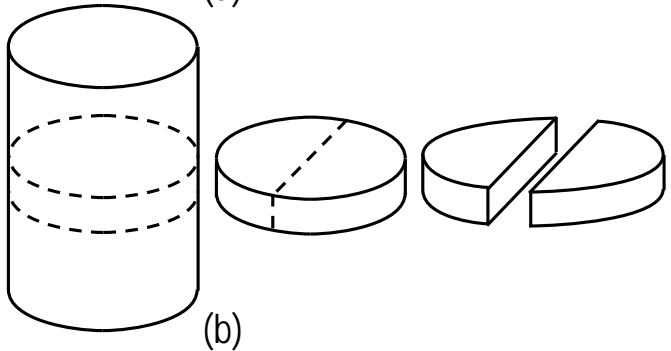

Figure 5. Specimens used for the $\left|\mathrm{E}^{*}\right|$ tests

Uniaxial compression and SCB tests with sinusoidal loads (haversine) were performed using a servo-pneumatic machine, developed at the Road Laboratory of the University of Rosario. Figure 6 shows an instrumented specimen for the uniaxial compression test. The $\left|E^{*}\right|$ measurements were determined for 7 frequencies $(5,4,2,1,0.5,0.25$ and $0.10 \mathrm{~Hz}$ ) and 5 temperatures $\left(0,10,20,30\right.$ and $\left.40^{\circ} \mathrm{C}\right)$ in order to have a full viscoelastic characterization of the asphalt mixtures. At each temperature, the lowest load compatible deformation with the capability of the data acquisition system was used in order to avoid induced damage in the samples.

\section{Obtained results}

Figures 7 and 8 show a comparison of the average dynamic modulus results obtained for two replicates with the Uniaxial Compression $\left(\left|\mathrm{E}^{*}\right|_{\mathrm{UC}}\right)$ and the Semi-Circular Bending configuration $\left(\left|\mathrm{E}^{*}\right|_{\mathrm{SCB}}\right)$ at all the testing frequencies and temperatures in a logarithmic space for the two mixtures considered in this study. Also in these figures, the line of equality and other two lines corresponding to relative differences equal to $\pm 25 \%$ are included.

As can be observed, the data points are well distributed along the line of equality with the $\mathrm{R}^{2}$ equal to $99.3 \%$ for the AC30 mixtures and 99.0\% for the AM3 mixture showing that the SCB configuration could be used for the determination of $\left|E^{*}\right|$ with the same sensitivity than the uniaxial compression test. 


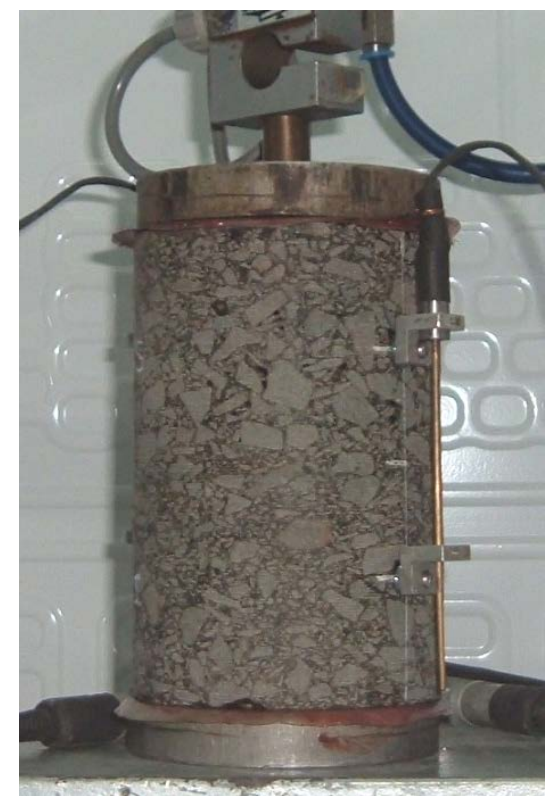

Figure 6. Specimen for the uniaxial compression test

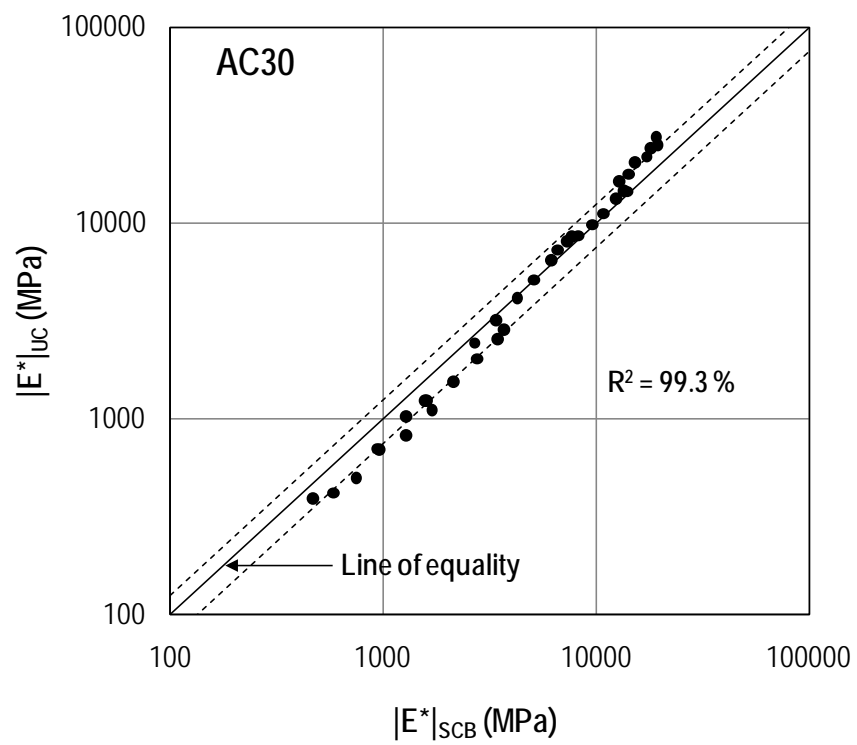

Figure 7. Comparison of dynamic modulus results for the AC30 mixture

In general, the relative differences between the $\left|\mathrm{E}^{*}\right|$ values measures with both procedures were lower than $25 \%$ and they are within the same orders of magnitude than others obtained from predictions of the dynamic modulus using predictive equations and models well accepted by the pavement community [29, 30].

These differences could be considered very acceptable since it was observed that replicate samples tested in the laboratory with the same experimental procedure, might exhibit a difference in the order of 20 to $30 \%$. In consequence, it could be established that both testing methodologies are measuring the same property within the acceptable range of difference to be applied for practical purposes in pavement design procedures.

Also during the dynamic modulus tests, the phase angle $\phi$ between loads and deformation was also measured. Figures 9 and 10 show the comparison of the average phase angles $\phi$ measured in the Uniaxial Compression tests ( $\left.\phi_{\mathrm{UC}}\right)$ and with the SCB geometry $\left(\phi_{\mathrm{SCB}}\right)$ for all the frequencies and temperatures and for the AC30 and AM3 mixtures respectively.

In these figures, the line of equality and a range of absolute differences equal to $\pm 5^{\circ}$ were also included. The obtained results are in well agreement with an $\mathrm{R}^{2}$ equal to $91.4 \%$ for the AC30 mixture and an $\mathrm{R}^{2}$ equal to $94.9 \%$ for the AM3 mixture. 
The $94 \%$ of the data points have absolute differences smaller than $5^{\circ}$ for the AC30 mixture and $86 \%$ for the AM3 mixture. However, it could be observed a slight tendency to measure smaller phase angles with the SCB configuration for the AM3 mixture.

Based on these experimental comparisons, the Semi-Circular Bending configuration with sinusoidal loads could be validated as a promising procedure for the determination of the dynamic modulus $\left|E^{*}\right|$ and the phase angle $\phi$ of asphalt mixtures.

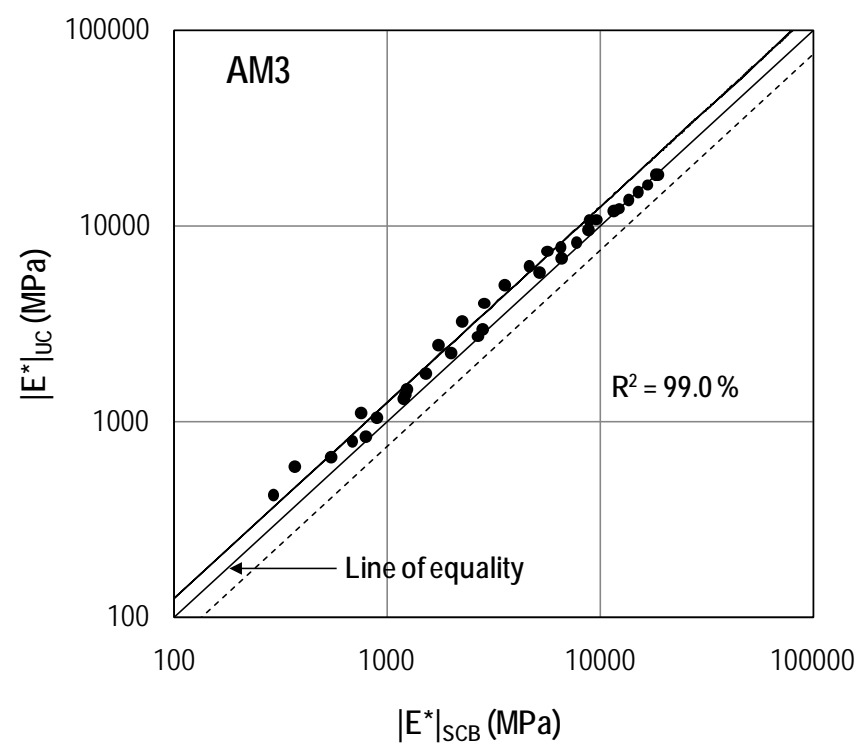

Figure 8. Comparison of dynamic modulus results for the AM3 mixture

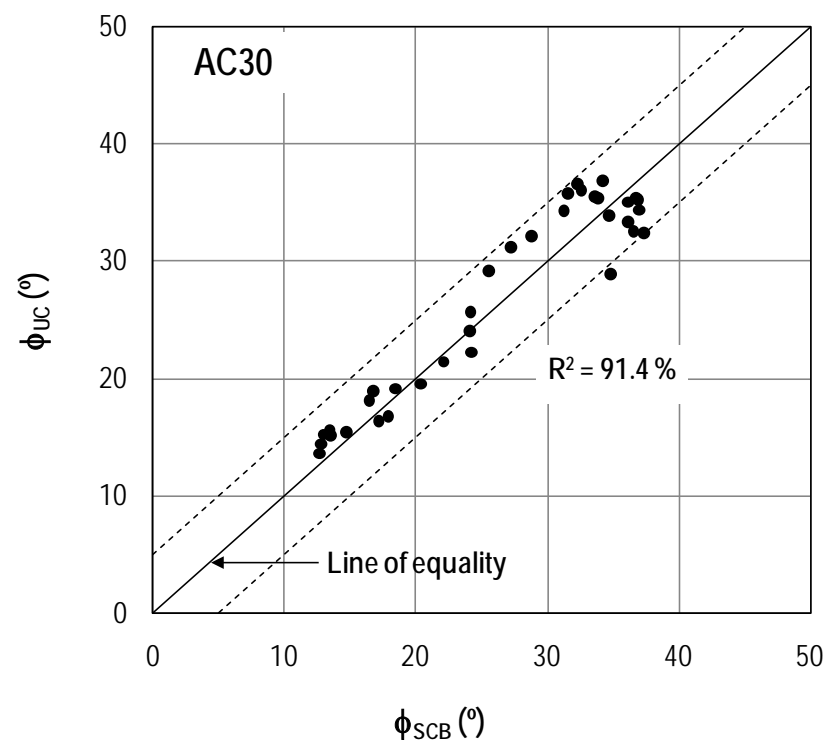

Figure 9. Comparison of phase angles for the AC30 mixture

\section{Rheological characterization}

\subsection{Viscoelastic master curves}

From the experimental results obtained for the two considered asphalt mixtures, single continuous curves for the dynamic modulus and the phase angle can be developed using the frequency-temperature superposition principle. 


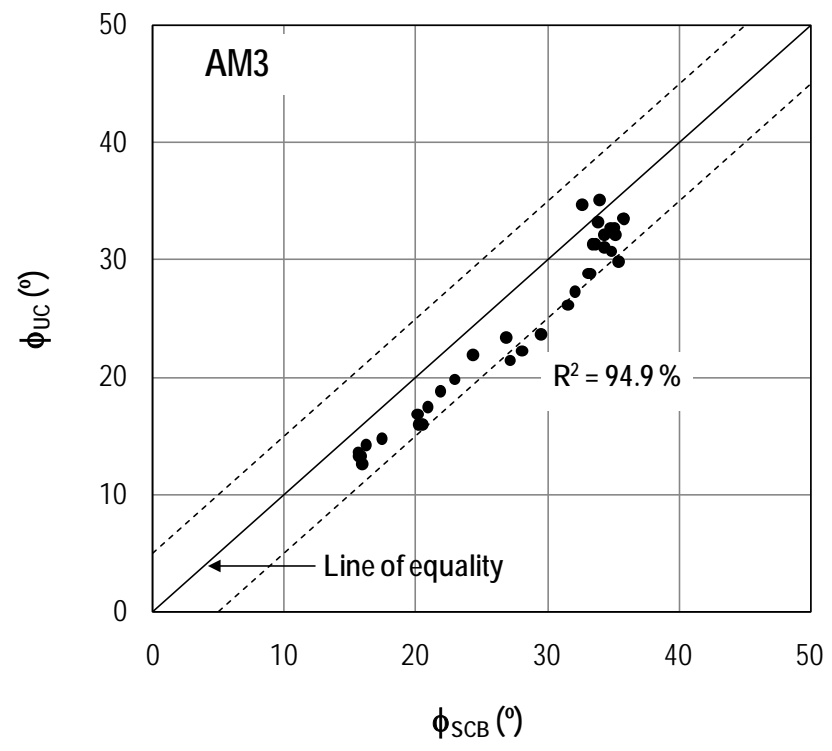

Figure 10. Comparison of phase angles for the AM3 mixture

To develop these curves, named Master Curves, $\left|E^{*}\right|$ values at various temperatures are horizontally shifted along the frequency axis in a logarithmic scale to form a single curve at a reference temperature TR. The equation for the shifted frequency in a logarithmic scale, known as the reduced frequency fR, is:

$$
f R=a T . T
$$

where $\mathrm{fR}$ is the Reduced frequency, $\mathrm{f}$ is the testing frequency and aT is the frequency-temperature shift factor.

The frequency-temperature shift factors for the reference temperature TR were modeled using an Arrhenius equation in the form:

$$
\ln (a T)=C \cdot\left[\frac{1}{T}-\frac{1}{T R}\right]
$$

where $\mathrm{C}$ is the Arrhenius parameter, $\mathrm{T}$ is the testing temperature $\left({ }^{\circ} \mathrm{K}\right)$ and $\mathrm{TR}$ is the reference temperature $\left({ }^{\circ} \mathrm{K}\right)$.

The $\left|\mathrm{E}^{*}\right|$ Master curves have been modeled according to a sigmoidal function in the form:

$$
\log (|E *|)=\alpha+\frac{\beta}{1+e^{[\gamma+\delta . \log (f R)]}}
$$

with $\alpha, \beta, \gamma$ and $\delta$, fitting parameters of the model.

The $\phi$ Master curves have been modeled using the same frequency-temperature shift factors obtained in the forming course of the $\left|\mathrm{E}^{*}\right|$ Master curves, according to an equation in the form of:

$$
\phi=\kappa \cdot\{\Gamma-\lambda\}^{\xi} \cdot e^{\{-\rho . \Gamma\}}
$$

with:

$$
\Gamma=\left[\log (f R)-\log \left(f R_{\min }\right)\right]
$$

where $\kappa, \lambda$, $\xi$ and $\rho$ are fitting parameters of the model and $\mathrm{fR}_{\min }$ is the minimum reduced frequency.

The fitting parameters were obtained simultaneously with the Arrhenius coefficient by minimizing the sum of the square errors between the experimental and model values using the Solver function in the Excel spreadsheet.

Figures 11 and 12 show the $\left|E^{*}\right|$ Master curves for the AC30 and the AM3 mixtures respectively. In these figures, the $\left|\mathrm{E}^{*}\right|$ Master curves for the average experimental results obtained in uniaxial compression and with the SCB configuration are compared.

For both mixtures, the Master curves obtained for both testing configurations are very similar with the same tendency. For the AC30 mixture, the SCB configuration trends to produce lower $\left|\mathrm{E}^{*}\right|$ values at high frequencies 
and higher $\left|\mathrm{E}^{*}\right|$ values at low frequencies. On the contrary, the SCB configuration trends to produce higher $\left|\mathrm{E}^{*}\right|$ values at high frequencies and lower $\left|\mathrm{E}^{*}\right|$ values at low frequencies for the AM3 mixture.

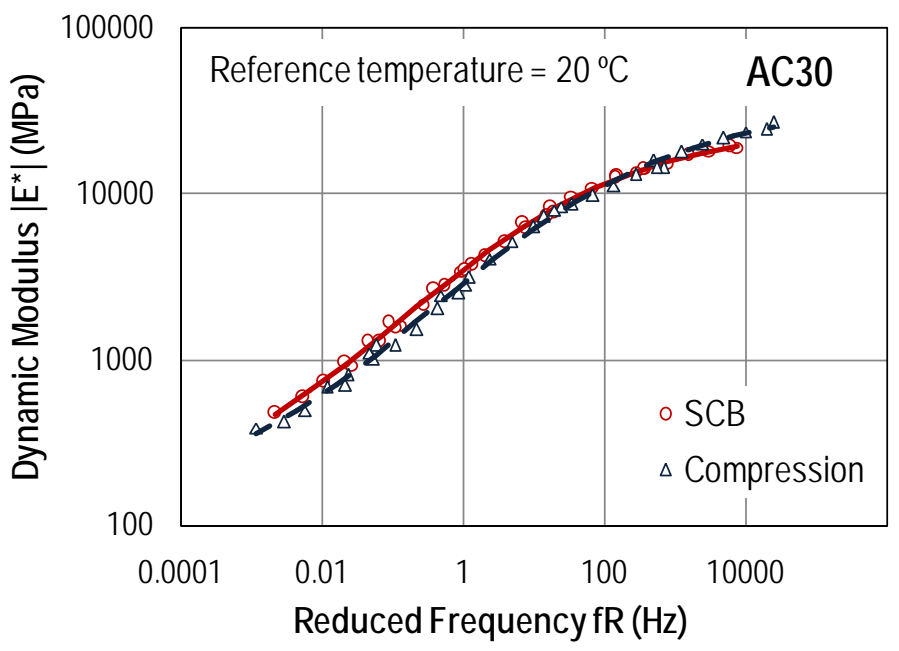

Figure 11. $\left|E^{*}\right|$ Master curve for the AC30 mixture

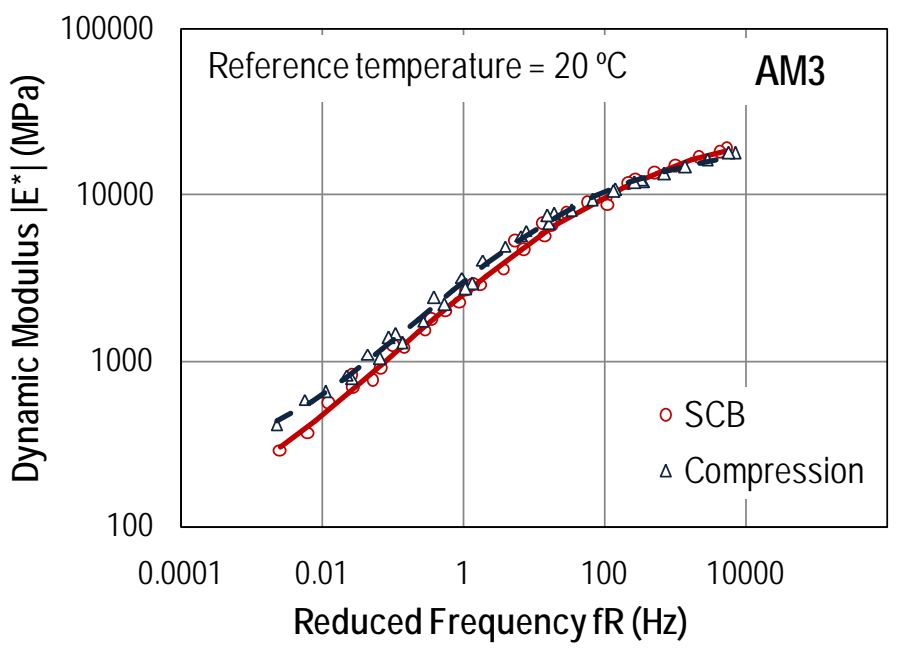

Figure 12. |E*| Master curve for the AM3 mixture

Figures 13 and 14 show the $\phi$ Master curves for the AC30 and the AM3 mixtures respectively. As in the previous figures, results obtained in uniaxial compression and with the SCB configuration are compared.

The observed tendency is consistent with findings reported by Biligiri et al. [31] and Geng et al. [32] with lower phase angles at high frequencies and higher phase angles at low frequencies. For both mixtures and with both testing configurations, phase angles ranging between 10 to $40^{\circ}$ were observed. The results obtained with the SBC and the uniaxial compression procedures are in very well agreement with differences smaller than $5^{\circ}$ for both asphalt mixtures considered in this evaluation.

For the AC30 mixture, the SCB configuration shows a tendency with higher values than the uniaxial compression configuration, mainly for the high frequency range.

As it was noted for the dynamic modulus, an opposite trend results for the AM3 mixtures with higher values for the SCB geometry compared to the phase angles measured in uniaxial compression.

Based on these observations it could be concluded that the SCB configuration is able to produce the same viscoelastic Master curves to be applied for practical purposes in the comparative evaluation of asphalt mixtures or in mechanistic pavement design procedures.

\subsection{Rheological models}

Values of the complex modulus $\mathrm{E}^{*}$ and phase angles $\mathrm{f}$ of the AC30 and AM3 mixtures were modeled using the Huet-Sayegh analogical model (H-S model). The coefficients describing the model are associated with simple mechanical behaviors, such as springs and parabolic dashpots, as illustrated in Figure 15 [33, 34]. 


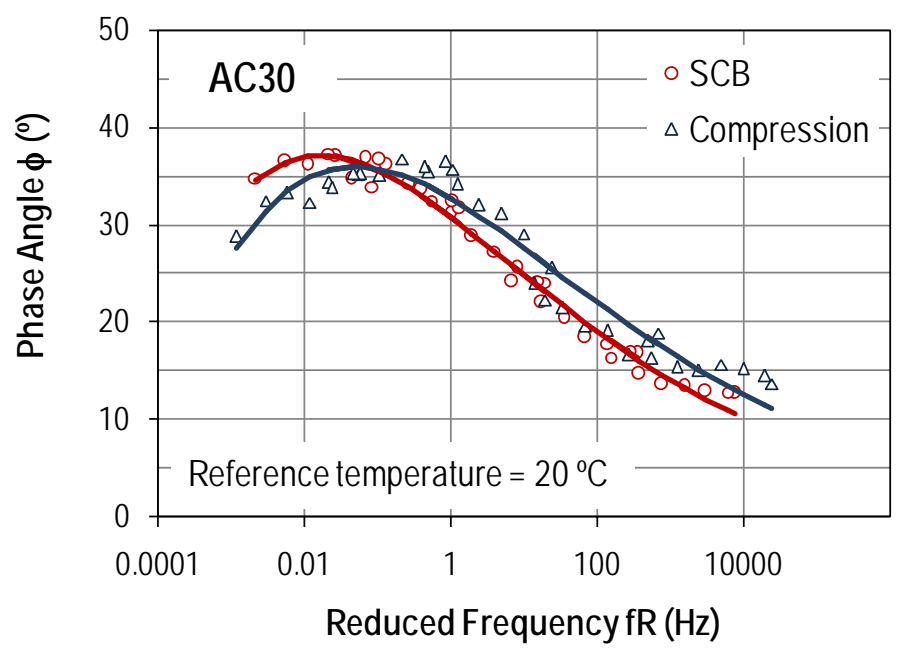

Figure 13. $\phi$ Master curve for the AC30 mixture

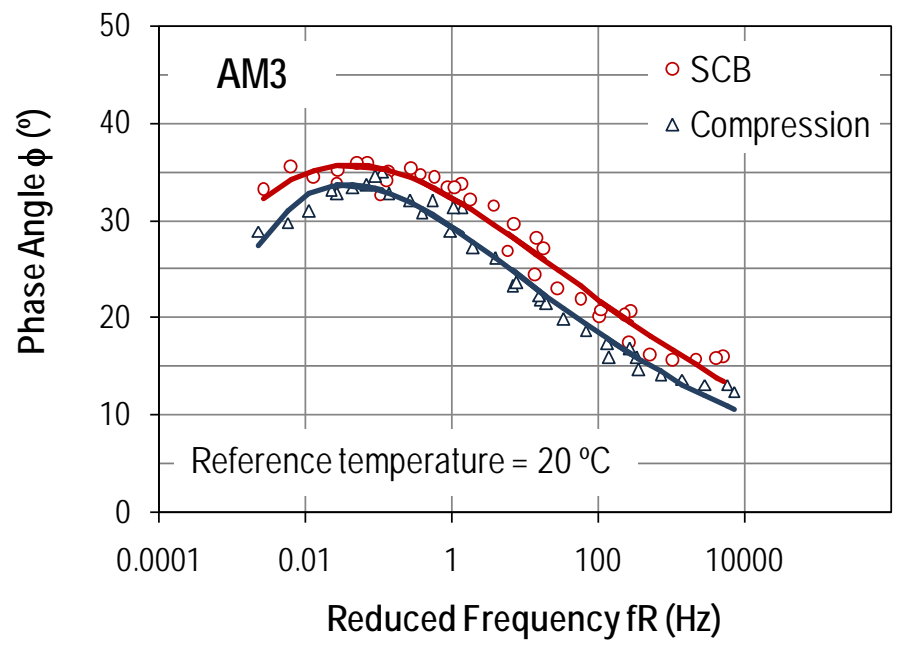

Figure 14. $\phi$ Master curve for the AM3 mixture

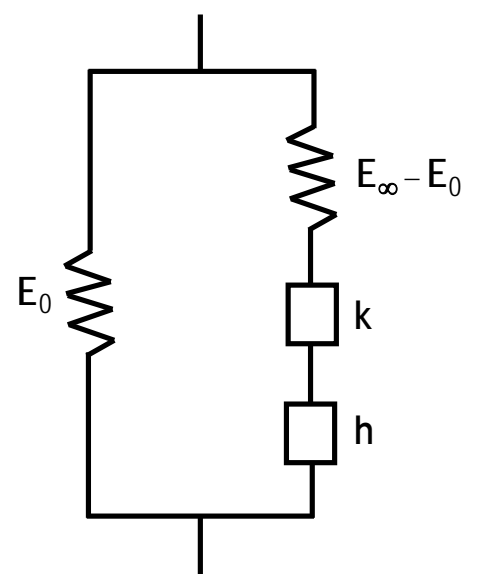

Figure 15. The Huet-Sayegh model

The model is a function of the angular frequency $\omega$ and the characteristic time $\tau$, adjusted for a reference temperature in accordance with the frequency-temperature superposition principle presented previously. The mathematical formulation of the H-S model is: 


$$
E^{*}=E_{0}+\frac{E_{\infty}-E_{0}}{1+\psi(i \omega \tau)^{-k}+(i \omega \tau)^{-h}}
$$

where $\mathrm{E} \infty$ is the instantaneous modulus for very short loading times, E0 is the static modulus for very long loading times, $\mathrm{i}$ is the imaginary number $\left(\mathrm{i}=(-1)^{1 / 2}\right)$, and $\mathrm{h}, \mathrm{k}$ and $\psi$ are parameters describing the model with $(1>\mathrm{h}>\mathrm{k}>0)$. A detailed description of the model can be found in a previous paper [35].

The characteristic time $\tau$ was calculated as:

$$
\tau=a T \cdot \tau_{0}
$$

where $\tau_{0}$ is the characteristic time at the reference temperature TR. The frequency-temperature shift factor aT was calculated using an Arrhenius equation as in the construction of the $\left|\mathrm{E}^{*}\right|$ Master curves in the form of:

$$
\ln (a T)=C_{H_{-} S} \cdot\left[\frac{1}{T}-\frac{1}{T R}\right]
$$

The coefficients $\mathrm{h}, \mathrm{k}$ and $\psi$ of the $\mathrm{H}$-S model, the Arrhenius parameter $\mathrm{C}_{\mathrm{H}-\mathrm{s}}$ and the characteristic time $\tau_{0}$ were adjusted using the least squares method between the predicted and measured values of the elastic (E1) and viscous (E2) components of $\mathrm{E}^{*}$ in the logarithmic space. The resulting parameters of the $\mathrm{H}-\mathrm{S}$ model and the characteristic time $\tau$ for the average experimental results obtained with the SCB and the uniaxial compression configuration for the two analyzed asphalt mixtures and for a reference temperature TR $=25^{\circ} \mathrm{C}$ are listed in Table 3 .

Table 3. Parameters describing the H-S model

\begin{tabular}{lcccc}
\hline & \multicolumn{2}{c}{ AC30 } & \multicolumn{2}{c}{ AM3 } \\
\cline { 2 - 5 } & SCB & Compression & SCB & Compression \\
\hline $\mathrm{E}_{\infty}(\mathrm{MPa})$ & 26573 & 35931 & 33601 & 22114 \\
$\mathrm{E}_{0}(\mathrm{MPa})$ & 121 & 119 & 39 & 164 \\
$\mathrm{C}_{\mathrm{H}-\mathrm{S}}$ & 21560 & 24990 & 21643 & 22542 \\
$\delta$ & 4.38 & 1.94 & 1.30 & 4.11 \\
$\mathrm{k}$ & 0.33 & 0.27 & 0.23 & 0.34 \\
$\mathrm{~h}$ & 0.64 & 0.51 & 0.46 & 0.61 \\
$\tau_{0}(\mathrm{~s})$ & $8.4110^{-2}$ & $4.3810^{-3}$ & $1.3210^{-3}$ & $7.8110^{-2}$ \\
\hline
\end{tabular}

The Black diagrams are graphs showing the variation of the phase angle $\phi$ as a function of the dynamic modulus $\left|E^{*}\right|$. In these plots, the frequency and the temperature are eliminated, which allows all the dynamic data to be presented in one graph. A smooth curve in a Black diagram is an indicator of the validity of the frequencytemperature superposition principle and a sign of the quality of the experimental values detecting the occurrence of inconsistencies in the rheological data caused by testing irregularities.

Figure 16 shows the Black diagram for the AC30 mixture while the Figure 17 shows the Black diagram for the AM3 mixture. In these figures, the average experimental values and the resulting $\mathrm{H}-\mathrm{S}$ models are displayed.

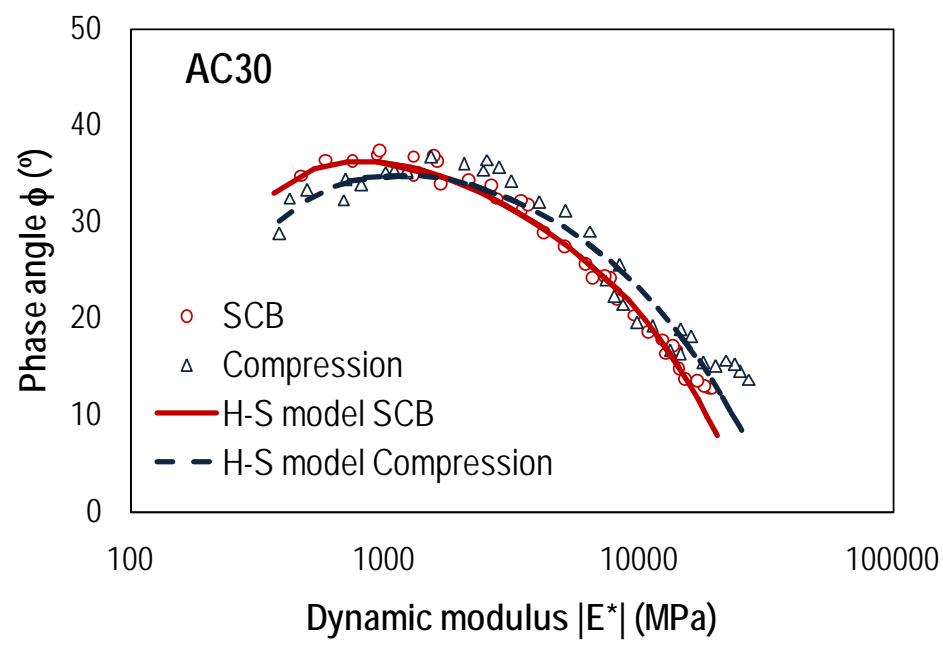

Figure 16. Black diagram for the AC30 mixture 


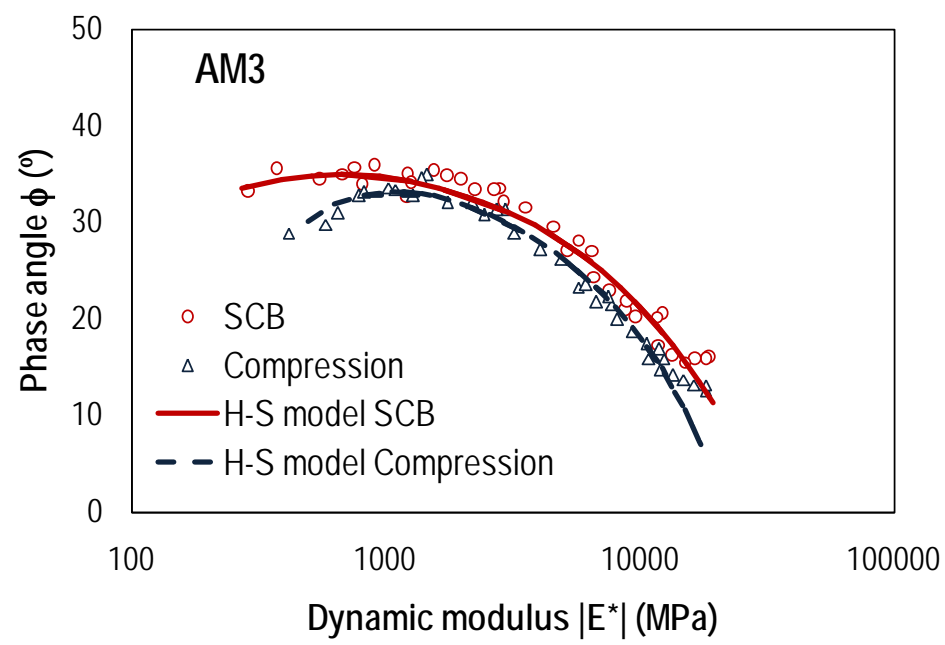

Figure 17. Black diagram for the AM3 mixture

For the asphalt mixtures considered in this paper, a good agreement for the results of the H-S models adjusted from the SCB and compression values was observed and with the same trends for both testing configurations.

Smooth curves have been drawn with the experimental results located in a narrow and tight band on both sides of these curves of the H-S model without any disruptions of the thermo-rheological simple behavior of the two asphalt mixtures and both testing configurations.

Based on these observations, the SCB geometry is able to produce experimental results that can be modeled with the same sensibility than others obtained with a more conventional testing procedures used for the rheological characterization of asphalt mixtures for practical purposes.

\section{Conclusions}

In this paper, the SCB configuration for the determination of the dynamic modulus $\left|\mathrm{E}^{*}\right|$ and the phase angle $\phi$ of two different asphalt mixtures has been investigated. For the specific adopted geometry (diameter of the rollers, spacing between rollers, gauge length, vertical location of the LVDT`s and thickness of the loading strip), an equation for the calculation of $\left|\mathrm{E}^{*}\right|$ was developed using a Finite Element model.

The instrumentation of the sample is relatively simple and any testing equipment suitable for dynamic modulus measurements with other standardized procedures could be easily adapted in order to accommodate the geometry proposed in this study.

Very well agreement has been found between results obtained with the uniaxial compression tests and the SBC configuration for both, the dynamic modulus $\left|\mathrm{E}^{*}\right|$ and the phase angle $\phi$ for a wide range of temperatures and frequencies considered in the experimental determinations with regression coefficients $\mathrm{R}^{2}$ higher than $90 \%$ in all cases.

The modeled Master curves for the dynamic modulus |E*| and phase angle $\phi$ were highly similar for both testing procedures and for the two asphalt mixtures. Also, the resulting Huet-Sayegh models adjusted from the average experimental results obtained with the SCB and in uniaxial compression were in good agreement.

Thus, it could be concluded that the SCB configuration has been validated as a simple and promising methodology for the characterization of the viscoelastic response of asphalt mixtures.

However, this last conclusion is supported by the results of two conventional asphalt mixtures commonly used in Argentina and, the applicability to other kind of mixtures (Open Porous Asphalt, SMA, mixtures containing RAP or waste by-products) should be verified.

As this study is part of an on-going research project at the University of Rosario, other experimental results will be considered in the near future in order to verify or re-calibrate this proposed procedure.

\section{References}

[1] Chong KP, Kuruppu MD. New specimen for fracture toughness determination for rock and other materials. International Journal of Fracture. 1984;26:59-62. 
[2] Lim IL, Johnston IW, Choi SK. Stress intensity factors for semi-circular specimens under three-point bending. Engineering Fracture Mechanics. 1993;44(3):363-382.

[3] Krans RL, Tolman F, Van de Ven MFC. Semi-circular bending test: A practical crack growth test using asphalt concrete cores. 3rd. International RILEM Conference on Reflective Cracking in Pavements. RILEM, Maastricht, The Netherlands.1996.

[4] Van de Ven M, Smit A, Krans R. Possibilities of a semi-circular bending test. Proceedings of the 8th International Conference on Asphalt Pavements. International Society for Asphalt Pavements, Seattle, Washington. 1997.

[5] Mull MA, Stuart K, Yehia A. Fracture resistance characterization of chemically modified crumb rubber asphalt pavement. Journal of Materials Science. 2002;37(3):557-566.

[6] Molenaar AA, Scarpas A, Liu X, Erkens SM. Semi-circular bending test; simple but useful?. Journal of the Association of Asphalt Paving Technologists. 2002;71: 794-815.

[7] Huang B, Shu X, Zuo G. Using notched semi circular bending fatigue test to characterize fracture resistance of asphalt mixtures. Engineering Fracture Mechanics. 2013;109:78-88.

[8] Wu Z, Mohammad LN, Wang LB, Mull MA. Fracture resistance characterization of superpave mixtures using the semi-circular bending test. Journal of ASTM International. 2005;2(3):127-141.

[9] Arabandi M, Ferdowsi B. Evaluating the semi-circular bending test for HMA mixtures. Journal IJE Transactions A. Basics. 2009; 22(1): 47-58.

[10] Li XJ, Marasteanu MO. Using semi circular bending test to evaluate low temperature fracture resistance for asphalt concrete. Experimental Mechanics. 2010;50(7):867-876.

[11] Shu X, Huang B, Vukosavljevic D. Evaluation of cracking resistance of recycled asphalt mixture using semicircular bending test. Paving Materials and Pavement Analysis (GSP 203). 2010.p. 58-65.

[12] Huang B, Shu X, Vukosavljevic D. Laboratory investigation of cracking resistance of hot-mix asphalt field mixtures containing screened reclaimed asphalt pavement. Journal of Materials in Civil Engineering. 2011;23(11):1535-1543.

[13] Li X, Marasteanu M. The fracture process zone in asphalt mixture at low temperature. Engineering Fracture Mechanics. 2010;77(7):1175-1190.

[14] Biligiri KP, Said S, Hakim H. Asphalt mixtures crack propagation assessment using semi-circular bending tests. International Journal of Pavement Research and Technology. 2012;5(4): 209-217.

[15] Elseifi MA, Mohammad LN, Ying H, Cooper III S. Modeling and evaluation of the cracking resistance of asphalt mixtures using the semi-circular bending test at intermediate temperatures. Road Materials and Pavement Design. 2012;13(sup1):124-139.

[16] European Committee for Standardization. EN 12697-44: Bituminous mixtures - Test methods for hot mix asphalt - Part 44: Crack propagation by semi-circular bending test. 2010.

[17] American Society of Testing Materials. ASTM D8044 - 16: Standard test method for evaluation of asphalt mixture cracking resistance using the semi-circular bend test (SCB) at intermediate temperatures. 2016.

[18] American Association of State and Highway Transportation Officials. AASHTO TP 105-13 Standard method of test for determining the fracture energy of asphalt mixtures using the semicircular bend geometry (SCB). 2013.

[19] Saha G, Biligiri KP. Stato-dynamic response analyses through semi-circular bending test: Fatigue life prediction of asphalt mixtures. Construction and Building Materials. 2017;150:664-672.

[20] American Association of State and Highway Transportation Officials. Standard method of test for determining dynamic modulus of hot-mix asphalt concrete mixtures. 2015.

[21] Martinez F, Angelone S. The indirect tensile test configuration in the determination of the complex modulus of asphalt mixtures. Proceedings of the 5th Eurasphalt and Eurobitume Congress; 2012.

[22] Zhang W, Drescher A, Newcomb DE. Viscoelastic analysis of diametrical compression of asphalt concrete. Journal of Engineering Mechanics. 1997;123: 596-603.

[23] Kim YR, Youngguk S, King M, Momen M. Dynamic modulus testing of asphalt concrete in indirect tension mode. Transportation Research Record: Journal of the Transportation Research Board. 2004;1891: 163-173.

[24] European Committee for Standardization. Bituminous mixtures - Test methods - Part 26: Stiffness. 2014.

[25] FGSV - Research Society for Roads and Traffic. Work instructions for the determination of the stiffness and fatigue behavior of asphalt mixtures with the indirect tensile test as an input variable. AL Sp-Asphalt 09. 2009 (In German).

[26] Witczak M, Mirza MW. Development of relation-ships to predict Poissons’s Ratio for paving materials. Interteam Technical Report for NCHRP 1-37A, University of Maryland, College Park, USA. 1999.

[27] Huang L, Cao K, Zeng M. Evaluation of semicircular bending test for determining tensile strength and stiffness modulus of asphalt mixtures. Journal of Testing and Evaluation. 2009;37(2):122-128.

[28] Giovanon O. Software for finite element calculations. Road Laboratory, Institute of Applied Mechanics and Structures, National University of Rosario. Argentina. 2007. 
[29] Andrei D, Witczak M, Mirza W. Development of a revised predictive model for the dynamic (complex) modulus of asphalt mixtures. Design Guide for New \& Rehabilitated Pavements. Appendix CC-4, D.(NCHRP Project 1-37A). National Research Council, Washington DC. 1999.

[30] Christensen DW, Pellinen TK, Bonaquist RF. Hirsch model for estimating the modulus of asphalt concrete. Journal of the Association of Asphalt Paving Technologists. 2003;72: 97-121.

[31] Biligiri KP, Kaloush K, Uzan J. Evaluation of asphalt mixtures' viscoelastic properties using phase angle relationships. International Journal of Pavement Engineering. 2010; 11(2): 143-152.

[32] Geng LT, Ren RB, Wang LZ, Gao CL. Master curves of dynamic modulus and phase angle for high modulus asphalt mixtures. Advanced Materials Research. 2011;243-249: 4226-4230.

[33] Huet C. Study of the viscoelastic behavior of bituminous mixes by method of impedance. [Doctoral dissertation]. Faculté des Sciences de l’Université de Paris, Paris. 1963. (In French).

[34] Pronk AC. The Huet-Sayegh model: A simple and excellent rheological model for master curves of asphaltic mixes. Proceedings of the Conference: R. Lytton Symposium on Mechanics of Flexible Pavements. American Society of Civil Engineers, Baton Rouge, Louisiana, USA. 2005.

[35] Cauhape Casaux M, Angelone S, Martinez F. Evaluation of the viscoelastic behavior of asphalt mixtures by mechanical models. Proceedings of the XIX Iberoamerican Congress of the Bitumen. Medellin, Colombia. 2017. (In Spanish).

(c) 2020 by the author(s). This work is licensed under a Creative Commons Attribution 4.0 International License (http://creativecommons.org/licenses/by/4.0/). Authors retain copyright of their work, with first publication rights granted to Tech Reviews Ltd. 